\title{
Two 35 day clocks in Hercules X-1: evidence for neutron star free precession
}

\author{
R. Staubert ${ }^{1}$, D. Klochkov ${ }^{1}$, K. Postnov ${ }^{2}$, N. Shakura ${ }^{2}$, J. Wilms ${ }^{3}$, and R. E. Rothschild ${ }^{4}$ \\ 1 Institut für Astronomie und Astrophysik, Universität Tübingen, Sand 1, 72076 Tübingen, Germany \\ e-mail: staubert@astro.uni-tuebingen.de \\ 2 Sternberg Astronomical Institute, 13 Universitetskii pr., 119992 Moscow, Russia \\ 3 Dr. Karl Remeis-Sternwarte, Astronomisches Institut der Universität Erlangen-Nürnberg, Sternwartstr. 7, 96049 Bamberg, Germany \\ ${ }^{4}$ Center for Astrophysics and Space Sciences, University of California at San Diego, La Jolla, CA 92093-0424, USA
}

Received 4 August 2008 / Accepted 13 November 2008

\section{ABSTRACT}

\begin{abstract}
We present evidence for the existence of two $\sim 35$ day clocks in the Her X-1/HZ Her binary system. $\sim 35$ day modulations are observed 1) in the Turn-On cycles with two on- and two off-states and 2) in the changing shape of the pulse profiles which re-appears regularly. The two ways of counting the 35 day cycles are generally in synchronization. This synchronization did apparently break down temporarily during the long Anomalous Low (AL3), which Her X-1 experienced in 1999/2000, in the sense that there must have been one extra Turn-On cycle. Our working hypothesis is that there are two clocks in the system, both with a period of about $\sim 35$ days: precession of the accretion disk (the less stable "Turn-On clock") and free precession of the neutron star (the more stable "Pulse profile clock"). We suggest that free precession of the neutron star is the master clock and that the precession of the accretion disk is basically synchronized to that of the neutron star through a feedback mechanism in the binary system. However, the Turn-On clock can slip against its master when the accretion disk has a very low inclination, as is observed to be the case during AL3. We take the apparent correlation between the histories of the Turn-Ons, of the Anomalous Lows and of the pulse period evolution, with a $5 \mathrm{yr}$ quasi-periodicity, as evidence for strong physical interaction and feedback between the major components in the system. We speculate that the $5 \mathrm{yr}(10 \mathrm{yr})$ period is due either to a corresponding activity cycle of $\mathrm{HZ}$ Her or a natural ringing period of the physical system of coupled components. The question of whether free precession really exists in neutron stars is very important for understanding matter with supra-nuclear density.
\end{abstract}

Key words. stars: binaries: general - accretion, accretion disks - stars: neutron - X-rays: general - X-rays: binaries X-rays: individuals: Her X-1

\section{Introduction}

The binary X-ray pulsar Her X-1 shows a number of periodic modulations of its X-ray flux: the 1.24 s pulse period, the $1.70 \mathrm{~d}$ orbital period (through eclipses and the Doppler modulation of the pulse period), a $1.62 \mathrm{~d}$ dip period, and a $35 \mathrm{~d}$ super-orbital period. The last period is observed first through an on-off cycle with a $10 \mathrm{~d}$ Main-On and a $5 \mathrm{~d}$ Short-On, separated by two $10 \mathrm{~d}$ Off-states (Tananbaum et al. 1972), and second through a reproduced change in the shape of the $1.24 \mathrm{~s}$ pulse profile (Trümper et al. 1986; Deeter et al. 1998; Scott et al. 2000). With respect to these modulations, we argue that there are two $\sim 35 \mathrm{~d}$ clocks in the system that are generally synchronized, but that were observed to temporarily lose synchronization during the long Anomalous Low in 1999/2000. Anomalous Lows seem to appear quasi-periodically about every 5 yrs (Staubert et al. 2006) and are believed to be connected with episodes of a low tilt of the accretion disk, which is then blocking the line of sight to the $\mathrm{X}$-ray source.

The 35 day modulation of the X-ray flux is generally explained by the precession of the accretion disk, which regularly blocks the line of sight to the X-ray emitting regions near the magnetic poles of the neutron star (Gerend \& Boynton 1976; Schandl et al. 1994). With regard to the systematic variation in the shape of the X-ray pulse profiles, we follow Trümper et al. (1986), Shakura et al. (1998), and Ketsaris et al. (2000) in assuming that the responsible physical mechanism is free precession of the neutron star.

Free precession may appear as a fundamental physical property of rigid non-spherical spinning bodies. The simplest case is a spheroid with some small oblateness (a "two-axial" body) in which the axis of angular velocity is not aligned with any principle axis of inertia (e.g., Klein \& Sommerfeld 1910). It has been suggested as the underlying reason for the long-period variations, both in timing and spectral properties, observed in several neutron stars (Jones \& Anderson 2001; Cutler et al. 2002; Link \& Epstein 2001; Haberl et al. 2006). The candidate objects are mostly radio pulsars (including the Crab and Vela pulsars), the isolated X-ray pulsar RXJ0720.4-3125 (Haberl et al. 2006) and the accreting binary X-ray pulsar Her X-1. The existence of free precession in neutron stars and its consequences for our understanding of the physics of the interior of neutron stars is extensively discussed in the literature (Anderson \& Itoh 1975; Shaham 1977; Alpar \& Ögelman 1987; Sedrakian et al. 1999; Wasserman 2003; Levin \& D’Angelo 2004; Link 2007). Recently, Link (2007) has emphasized that the question of the reality of free precession in neutron stars has strong implications for our understanding of the properties of matter at supra-nuclear densities.

In Her X-1 the case for free precession is as longstanding (Brecher 1972; Trümper et al. 1986; Shakura et al. 1998) as it 


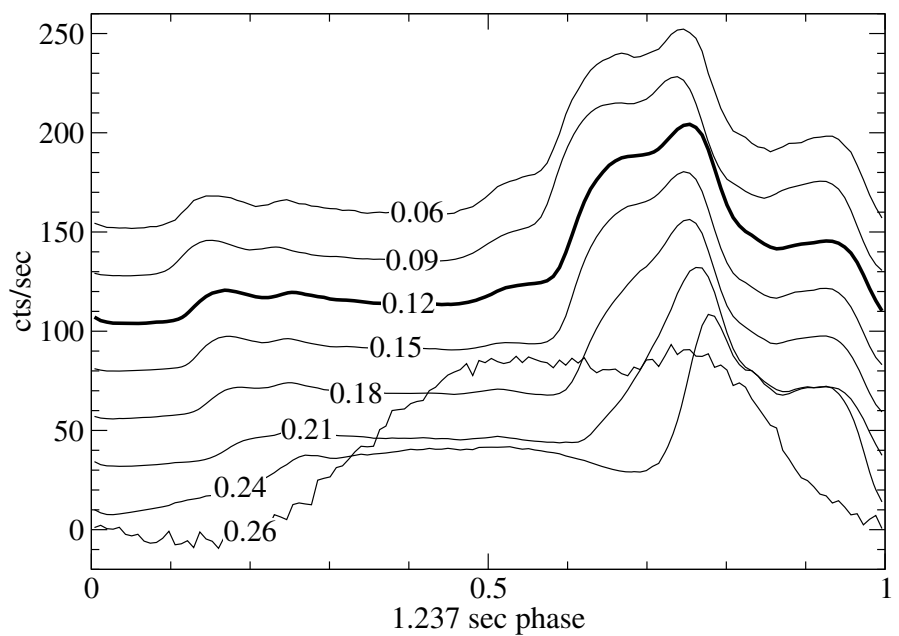

Fig. 1. Pulse profiles of Her X-1 as observed by RXTE/PCA (3-20 keV) during the Main-On of November 2002 (35 d cycle No. 323 ${ }^{1}$, according to the pulse profile counting) as a function of $35 \mathrm{~d}$ phase. For better visibility the profiles are scaled to a common amplitude and shifted against each other according to their $35 \mathrm{~d}$ phase. The profiles were generated using 128 phase bins and the curves are the straight line connection between adjacent data points (which are not shown).

is criticized on various grounds (e.g., Bisnovatyi-Kogan et al. 1989). Here we provide new observational clues of a very stable clock that governs the regularly re-appearing pulse profiles. We identify the stable clock with free precession of the neutron star and suggest that the accretion disk with its rather unstable Turn-On clock is a slave to the neutron star on long time scales through a closed loop physical feedback in the binary system.

\section{Pulse profile cycle counting}

The shape of the pulse profiles of the $1.24 \mathrm{~s}$ X-ray pulsations is known to vary in several different ways, e.g., as a function of energy (Gruber et al. 1980) and as a function of $35 \mathrm{~d}$ phase (Trümper et al. 1986; Soong et al. 1990; Deeter et al. 1998; Scott et al. 2000). Here, we are concerned with the latter, the systematic variation with $35 \mathrm{~d}$ phase. In Fig. 1 we show the result of our analysis for the Main-On state of 2002 November observed by RXTE. Our working hypothesis is that this modulation is due to a precessing neutron star, which forces our viewing angle towards the X-ray emitting polar cap region of the neutron star to vary with the phase of the neutron star precession.

Using observations by RXTE from 1996 until 2005, we verified that the shape of the pulse profiles is reproduced every $\sim 35$ days. A careful timing analysis was performed of all archived RXTE data on Her X-1 and pulse profiles were generated by folding with the measured pulse periods. Figure 1 shows a set of pulse profiles (PCA, 3-20 keV) from $35 \mathrm{~d}$ cycle No. $323^{1}$ (Nov. 2002) for eight different $35 \mathrm{~d}$ phases. The variation of the pulse shape is evident. Using a profile from cycle No. 257 at $35 \mathrm{~d}$ phase 0.12 as a template (quite similar to the phase 0.12 profile of cycle No. 323, highlighted in Fig. 2), we performed a comparison to profiles from other cycles. For this purpose all profiles were normalized using the amplitude of the main peak. Then the profiles were aligned in pulse phase. Within one $35 \mathrm{~d}$ mainon this is assured by phase connection, the alignment of profiles from different $35 \mathrm{~d}$ cycles was done using the "sharp edge" at the

1 The counting of $35 \mathrm{~d}$ cycles follows the convention used by Staubert et al. (1983): (O-C) = 0 for cycle No. 31 with turn-on near JD 2442410.

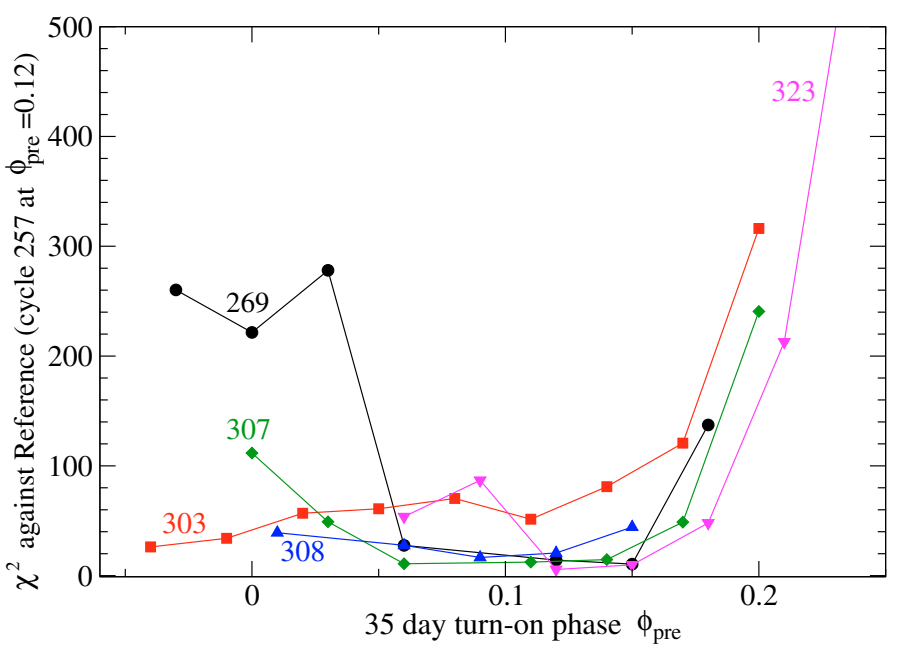

Fig. 2. Comparison of pulse profiles observed in a number of 35 day cycles at different 35 day phases to a template profile of cycle No. 257 at phase 0.12 . The match to the template profile is measured by the respective $\chi^{2}$-value.

decay of the shoulder to the right of the main peak, which was found to be the sharpest and most stable feature of the Her X-1 pulse profile. Then the difference between the count rates in the 128 phase bins were taken, squared, and summed. This $\chi^{2}$-sum is taken as a quantitative measure for the "matching" of the individual profiles to the template. There are profiles from 16 different 35 d cycles taken by $R X T E$ over 8.8 years. However, only in 7 cycles are pulse profiles for 4 or more different $35 \mathrm{~d}$ phases available. In Fig. 2 the $\chi^{2}$-values are plotted for those 5 cycles for which profiles at 7 or more different phases are available. Each data point corresponds to a pulse profile generated from data of one complete day of RXTE observations. The center time of the observing interval is translated into a $35 \mathrm{~d}$ phase (using a constant period of 34.85 days). The minimum in $\chi^{2}$ for the different cycles is generally found around $35 \mathrm{~d}$ phase 0.12 (the phase of the template), demonstrating that the profiles are repeating regularly. The $\chi^{2}$ comparison was repeated with three other templates (from different $35 \mathrm{~d}$ cycles and different phases), yielding the same result. This establishes a method of cycle counting that is based solely on the shape of the pulse profile.

\section{Turn-On cycle counting}

The second method of cycle counting uses the well-established $35 \mathrm{~d}$ modulation of the X-ray flux (Giacconi et al. 1973): the $\mathrm{X}$-ray flux increases sharply at Turn-On, reaching a maximum of the Main-On which lasts for a few days, before fading slowly into the first minimum. After that, a second, substantially lower maximum, the Short-On, emerges for a few days, after which a second minimum concludes the cycle (see Klochkov et al. 2006). It is generally assumed that the modulation is due to shading by the precessing accretion disk (Gerend \& Boynton 1976). There is a Turn-On roughly every 35 days. However, this clock is not very accurate: the length of a particular cycle may be longer or shorter than the previous one by $\sim 0.85$ days $\left(=P_{\text {orb }} / 2\right)$.

The irregularity of the Turn-On clock is demonstrated by the $(\mathrm{O}-\mathrm{C})$ diagram (Fig. 3, lower panel). Here the difference between the observed turn-on time $(\mathrm{O})$ and the calculated turn-on time (C) is plotted against time (Staubert et al. 1983; Staubert et al. 2000; Still \& Boyd 2004; Staubert et al. 2006). 


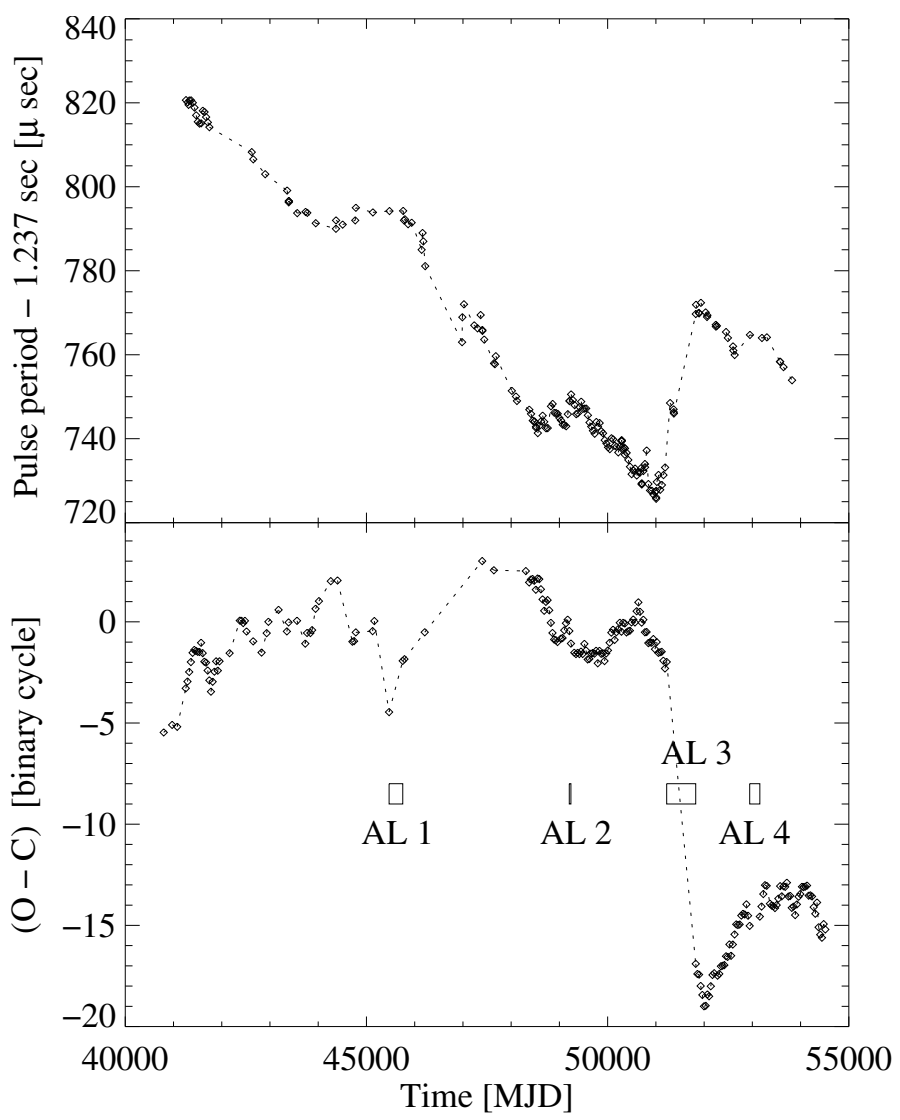

Fig. 3. Pulse period and Turn-On histories of Her X-1 (an update of Fig. 1 of Staubert et al. 2006).

To calculate the turn-on time, a constant period of $34.85 \mathrm{~d}$ was used (equal to $20.5 \times P_{\text {orb }}$, with $P_{\text {orb }}=1.700 \mathrm{~d}$ ). Figure 3 is our latest update of this diagram. If $(\mathrm{O}-\mathrm{C})$ is measured in units of $P_{\text {orb }}$, all data points fall more or less on horizontal lines (spaced by $0.5 \times P_{\text {orb }}$ ), due to the observed fact that the Turn-Ons occur close to binary phases 0.25 or 0.75 . Staubert et al. (1983) (at a time when only data just beyond the first Anomalous Low AL1 - were available) had postulated that the change in $(\mathrm{O}-\mathrm{C})$ from one cycle to the next should be either 0 or +1 or -1 in units of $P_{\text {orb }} / 2$ (corresponding to a cycle length of 20.5 or 21 or 20 binary periods). Even though the short-term development was successfully modeled by a random-walk process, they argued for the possibility that the global long-term development of the diagram might be nearly flat, indicating some sort of a "backdriving force" that would prevent the wandering off to one or the other side. These assumptions have been found to hold until the dramatic event of the Anomalous Low of 1999/2000 (AL3).

Figure 3 demonstrates that the Turn-On clock is quite noisy, with additional quasi-periodic variations on a 5 year time scale. (O-C) correlates with the appearance of the Anomalous Lows (AL), and it also strongly correlates with the neutron star's spin period (Staubert et al. 2006, 2008b).

\section{Difference between pulse profile counting and Turn-On counting}

The two ways of cycle counting are normally consistent with one another. However, during the long Anomalous Low in 1999/2000 (AL3 in Fig. 3) the synchronization was apparently lost.

In Fig. 5 we plot the absolute times of the $\chi^{2}$-minima (see Figs. 1 and 2) found when the pulse profiles observed in the

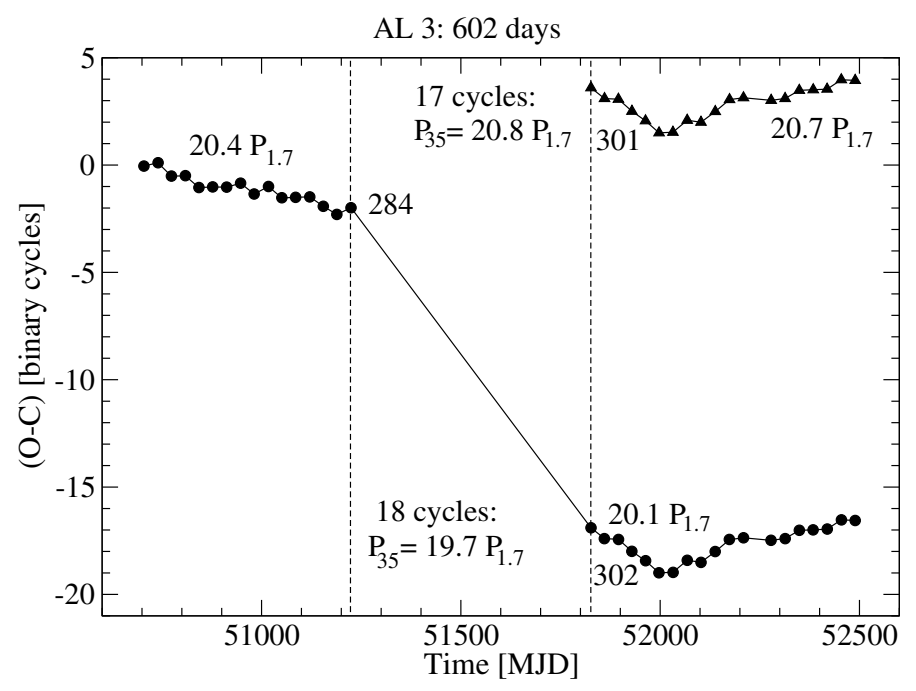

Fig. 4. The Anomalous Low AL3 must be bridged by 18 (not 17) TurnOn cycles: from cycle No. 284 to cycle No. 302.

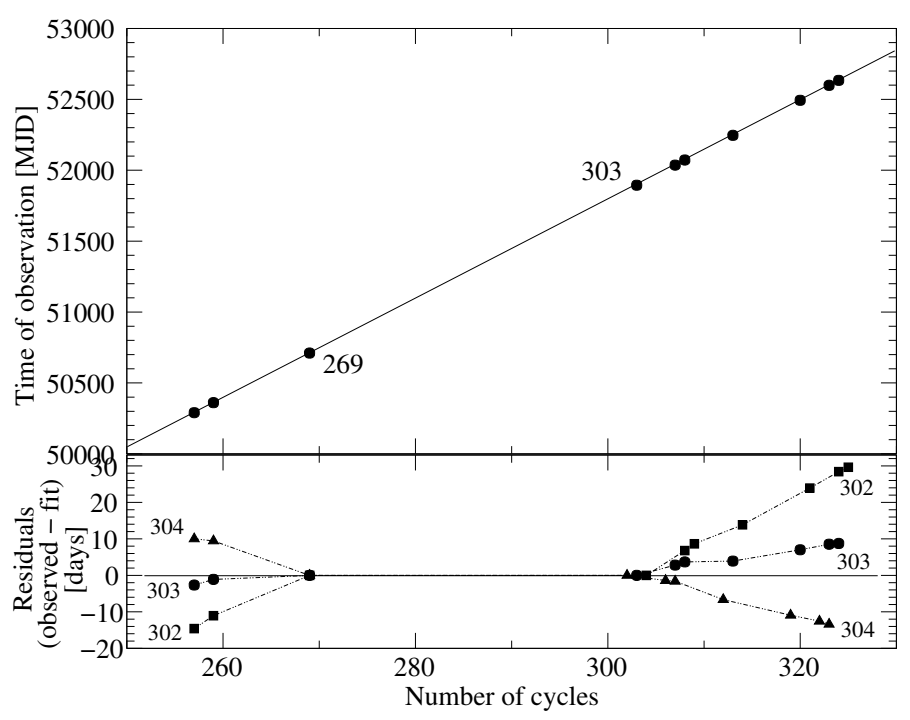

Fig. 5. Cycle counting by pulse-profile fitting. Upper panel: absolute times of $\chi^{2}$-minima obtained through template fitting versus cycle number (assuming that the gap is between cycle Nos. 269 and 303). Lower panel: residuals against the straight line connection of cycles 269 and 303 (center curve) or for the two cases where the first cycle observed after AL3 is called 302 or 304, respectively. The uncertainties in time are generally less than $0.5 \mathrm{~d}$ (smaller than the size of the symbols).

respective Main-Ons were compared to the template profile of cycle No. 257, against the cycle number (from pulse profile counting). The last Main-On before AL3 in which good pulse profiles were obtained belongs to cycle No. 269, and the first Main-On after AL3 is cycle No. 303. The straight line in Fig. 5 (upper panel) is the connection between the data points of cycle No. 269 and cycle No. 303. Dividing the difference in the corresponding absolute times for the $\chi^{2}$-minima by 34 (=303-269) leads to a cycle length of $34.95 \pm 0.01 \mathrm{~d}$. The lower panel in Fig. 5 shows the residuals of the data points to the straight line: the center curve is for the proposed cycle counting (269/303). Any different counting to bridge the gap (e.g., 269/302 or 269/304) can be ruled out, Fig. 5 establishes that the reference pulse shape (our template at $35 \mathrm{~d}$ phase 0.12 ) does regularly repeat and that the corresponding observing times can be associated with unique cycle numbers. A linear fit to the data point in the upper panel of 
Fig. 5 yields a mean period of $34.98 \pm 0.01 \mathrm{~d}\left(=20.58 \cdot P_{1.7}\right)$ from pulse profile counting. (Repeating the exercise with three other template profiles yields the same result within uncertainties.)

Figure 4 shows the $(\mathrm{O}-\mathrm{C})$ diagram around AL3. Turn-Ons are not observed during the AL, since the source is strongly obscured for a total of 602 days. Assuming that the physics behind this counting, that is the precession of the accretion disk, is continuing during the AL, one can estimate the number of cycles during AL3. There are two possible solutions: 17 cycles with a mean period $20.8 \cdot P_{1.7}$ or 18 cycles with a mean period of $19.7 \cdot P_{1.7}$. We conclude that there must have been 18 cycles, not 17 , because of the following arguments.

1. Before AL3 the mean period was low $\left(20.4 \cdot P_{1.7}\right)$, as it is always the case when going into an AL (Coburn et al. 2002). Coming out of AL3, the mean period of the next 6 cycles is even lower $\left(20.1 \cdot P_{1.7}\right)$. It is unreasonable to assume that the period was longer in between.

2. The strong long-term anti-correlation between $(\mathrm{O}-\mathrm{C})$ and the neutron star's spin and the observed dramatic spin-down during AL3 requires that $(\mathrm{O}-\mathrm{C})$ needs to drop during AL3 (Staubert et al. 2006), equivalent to a short period.

3. It is believed that the Anomalous Low is caused by the blocking of the line of sight to the X-ray emitting region by the accretion disk due to a decreased tilt of the disk with respect to the orbital plane.

The decrease in tilt angle is likely due to a reduced mass transfer rate, leading to a reduced momentum of the stream onto the disk, which is equivalent to a reduced breaking of the precession of the disk and consequently a higher precession frequency (Klochkov et al. 2006).

We conclude that during AL3 the accretion disk did one extra cycle in comparison to the regular cycle counting using the pulse profiles.

\section{Summary of observational facts}

Before entering the discussion, we summarize the observational facts relevant to our conclusion about the reality of free precession of the neutron star in Her X-1.

1. The pulse profile shape varies with $35 \mathrm{~d}$ phase and repeats regularly. The observations by $R X T E$ over $\sim 9$ yrs establish a stable clock with a period of $(34.98 \pm 0.01) \mathrm{d}$.

2. The Turn-On clock is rather unstable: it shows a quasiperiodic variation in $(\mathrm{O}-\mathrm{C})$ with $\mathrm{a} \sim 5 \mathrm{yr}$ period and an amplitude of $\sim \pm 3 P_{\text {orb}}$, with "substructure" and additional noise, and a large step in (O-C) during the 1999/2000 Anomalous Low (AL3), in correlation with a dramatic spindown (Fig. 3).

3. Anomalous Lows (ALs) appear quasi-periodically every $\sim 5$ yrs, in correlation with minima in $(\mathrm{O}-\mathrm{C})$.

4. The Turn-On history $(\mathrm{O}-\mathrm{C})$ is strongly correlated to the pulse period evolution (Fig. 3, and Staubert et al. 2006).

5. The counting of $35 \mathrm{~d}$ cycles by Turn-Ons, the $(\mathrm{O}-\mathrm{C})$ diagram, is generally synchronized (with deviations of up to $\sim \pm 3 P_{\text {orb }}$ ) to the counting of $35 \mathrm{~d}$ cycles using pulse profiles.

6. During the long AL of 1999/2000 (AL3), the Turn-On period was so low such that one extra cycle was done compared to the pulse profile counting.

\section{Discussion and conclusions}

We conclude that there are two clocks in Her X-1, both with a period of about $35 \mathrm{~d}$ : precession of the accretion disk and free precession of the neutron star. The precessing outer rim of the accretion disk regularly blocks the line of sight to the X-ray emitting polar caps of the neutron star, thereby producing the Turn-On cycle. The free precession of the neutron star is responsible for the orientation of the beamed X-ray emission, thereby producing the periodic modulation of the shape of the observed pulse profiles.

The two clocks are so similar in period because of synchronization due to strong feedback in the system. (It would seem unreasonable to assume that the two clocks have nearly the same frequency purely by chance.) We propose that free precession of the neutron star is the master clock which is rather stable. (It could however change rapidly due to a change in the shape of the neutron star, e.g., as a result of a star quake.) The accretion disk precession is observed to be a noisy clock with some systematic variations. There are quite a number of torques acting on the accretion disk: the tidal force from the optical star, the internal viscous force, dynamical forces due to the impact of the accretion stream and the illumination by the X-ray beam, and, finally, the neutron star magnetosphere interacting with the inner edge of the disk. These forces collectively produce the precession (as well as the tilt and the warp) of the disk (Shakura et al. 1998; Klochkov et al. 2006). We consider the tidal force and the dynamical forces to be the dominating ones. In the absence of dynamical forces, the precession would be much faster, with a period around $15 \mathrm{~d}$ (Shakura et al. 1999).

So the question is: how does the neutron star with its inherent period of free precession of $35 \mathrm{~d}$ manage to "enslave" the accretion disk? We believe that the critical parameter for the closed loop feedback in the system is the rate of mass transfer from the optical star. The surface of HZ Her facing the neutron star is illuminated and heated by the neutron star's X-ray emission, enhancing the mass transfer. However, the heating is not constant and uniform because the accretion disk blocks part of the X-ray beam and modulates (spatially and temporally) the heating of the optical star's surface according to its precessional motion. A first loop may be the following. The inner part of the accretion disk follows the free precession of the neutron star (because of the magnetospheric interaction), and the shadowing of the optical star from the X-ray beam follows the movement of the inner disk, modulating the mass transfer rate with the period of the neutron star free precession. The accretion stream hits the accretion disk applying a force to slow down the disk's precession and to influence its tilt, as shown by numerical simulations (Shakura et al. 1999; Klochkov et al. 2006). A variable tilt of the outer disk results again in a variable shading of the optical star. Finally, the variable mass transfer rate will eventually (after a viscous time scale) show up in a variable mass accretion rate that governs the X-ray luminosity. The X-ray luminosity, on the other hand, is crucial for the heating of the optical star as well as for the illumination of the outer parts of the accretion disk where coronal winds and torques may be produced (Schandl et al. 1994). With the variable heating of the optical star by the X-ray beam, both through variable shading and through variable X-ray flux, the loop is closed.

Also, the strong correlation between the $(\mathrm{O}-\mathrm{C})$ diagram and the pulse period evolution (Fig. 3 and Staubert et al. 2006) can be understood within this model. Any enhanced mass accretion rate (because of an enhanced mass transfer rate) will result in an enhanced angular momentum transfer, hence in a spin-up.

We propose that the described physical couplings provide strong feedback mechanisms in the Her X-1/HZ Her binary system that establish a delicate equilibrium in the whole system. We assume that the physical coupling described above is strong 
enough to lock the precession of the outer accretion disk to that of the neutron star. (This may not be possible if the natural frequencies of the precession of the neutron star and that of the accretion disk were different by much more than the estimated factor of about 2.) Due to the large number of forces acting on the accretion disk (probably all of them being subject to noise) one may understand that the synchronization between the two clocks is not perfect: First, there is the modulation of the TurnOn times. We know now that a random walk is not the right model for this modulation. There seems to be just random noise superimposed onto the quasi-periodic up and down in $(\mathrm{O}-\mathrm{C})$. Over long times the deviations are limited to $\sim \pm 3 P_{\text {orb }}$. In our current model we would now interpret the "back-driving force" postulated by Staubert et al. (1983) as being due to the coupling of the accretion disk to the neutron star. Second, the dramatic event observed during AL3, in which the accretion disk showed a low tilt and a fast precession, could then be viewed as extreme behavior, demonstrating that the accretion disk has a "life of its own", and it is able to temporarily escape the slaving by the neutron star. It seems, however, that the equilibrium is re-established rather quickly. In quoting a $35 \mathrm{~d}$ cycle no. for the time after AL3, one has to clearly state what method of cycle counting it refers to: pulse profile counting or Turn-On counting, the latter is advanced by one cycle.

We would also like to draw attention to the fact that the (O-C) diagram (Figs. 3 and 4) has - averaged over time scales $>15 \mathrm{yrs}-$ a positive slope from the time of discovery of the source until today, if pulse profile counting is used. In this case the diagram continues after AL3 with the upper right hand curve in Fig. 4, corresponding to the solution with 17 neutron star cycles inside AL3. A linear fit to all data of the $(\mathrm{O}-\mathrm{C})$ diagram in pulse profile counting yields a mean cycle duration of $(34.8791 \pm 0.0001) \mathrm{d}$, which we associate with the long-term average period of the neutron star precession. Using only data after AL2, we find an average period of $(34.9681 \pm 0.0003) d$, quite close to the period of $(34.98 \pm 0.01) \mathrm{d}$, found from pulseprofile fitting (over a similar observational period). The quoted uncertainties, however, are only statistical uncertainties; for the true physical uncertainties, one would have to add systematic uncertainties due to the irregular modulation over the limited time base and the non-uniform sampling of $(\mathrm{O}-\mathrm{C})$. We thus refrain from speculating about variation in the period over time. We take the above finding as independent support for the already reached conclusion that the precession of the accretion disk follows that of the neutron star on long time scales. We predict that the mean upward trend in $(\mathrm{O}-\mathrm{C})$ will continue in the future.

The key feature of our model is that free precession of the neutron star is responsible for the observed long-term stability of the $35 \mathrm{~d}$ cycle (both, the regular re-appearance of pulse profiles as well as the long-term turn-on history). Unlike in isolated neutron stars, where free precession is damped by dissipative processes, the free precession of the old neutron star in Her X-1 can be sustained for long times by the accretion feedback loop described above, which may lead to quite different properties. We also note that Lamb et al. (1975) have already concluded that phase dependent torques are capable of exciting (or damping) large amplitude neutron star wobble.

With regard to the apparent quasi-periodic $5 \mathrm{yr}(10 \mathrm{yr})$ modulation in $(\mathrm{O}-\mathrm{C})$, seen in correlation with the pulse period evolution and the appearance of the Anomalows Lows, we have no definite answer. We see two possibilities (Staubert et al. 2006): either the modulation is due to an "activity cycle" of HZ Her changing the mass transfer rate (see also Still \& Jurua 2006), or the $\sim 5 \mathrm{yr}$ may represent a natural ringing frequency of a system of several coupled physical components.

We would like to address a final question: are shifts in pulse arrival time observed, which are expected to occur for a precessing pulsar? A decomposition analysis of high-quality pulse profiles observed with RXTE using eight Gaussian components shows that the main peak (as well as other peaks) is (are) systematically varying in amplitude and in relative phase (e.g., with respect to the well-defined minimum). A quantitative description is in preparation. In addition, we are making progress in modeling the changing shape of the pulse profiles. First results, assuming a spot-like emission region at each of the two magnetic poles and additional arc-like emission structures around the poles, were published by Wilms et al. (2003) and again reported by Postnov (2004). In the case of a freely precessing neutron star radiating like a pulsar, systematic variations in the observed period and pulse phase with $35 \mathrm{~d}$ phase are expected (Ruderman 1970; Shakura 1988; Postnov et al. 1991; Bisnovatyi-Kogan \& Kahabka 1993), with a maximum relative change in period on the order $10^{-6}$. Any change in pulse period will result in a shift in the pulse arrival time. Unfortunately, such shifts are observationally indistinguishable from shifts resulting from period variations due to accretion torque changes. The pulse period history shown in Fig. 3 (upper panel) shows strong and frequent period variations on time scales ranging from $18 \mathrm{~d}$ to beyond $1000 \mathrm{~d}$. The strongest variations reach $\mathrm{d} P / \mathrm{d} t$-values of nearly $\pm 3 \times 10^{-12} \mathrm{~s} \mathrm{~s}^{-1}$ (observed on the smallest time scales), corresponding to a relative change of $10^{-6}$ over a few days. In addition, detailed pulse arrival time analysis of RXTE data has shown that similar changes (of both signs) are found on time scales of a few days (see also Klochkov et al. 2008). Our six measured values do not show any correlation with $35 \mathrm{~d}$ phase. We attribute these to changes in mass accretion rate.

In summary, we conclude that our analysis does support the idea of free precession to be present in the neutron star of Her X-1. Our main line of arguments rest on the identification of two different $35 \mathrm{~d}$ clocks in this system: free precession of the neutron star (as the master clock) and precession of the accretion disk (which is quasi-synchronized to the neutron star for most of the time). Long-term shifts in pulse arrival time, as seen in radio pulsars, are principally non-observable because of the always present accretion torque noise and resulting pulsefrequency variations. However, the observed systematic relative shifts of structures in the pulse profile with corresponding changes in pulse width and amplitude as a function of precessional phase are reminiscent of precessing radio pulsars. Link (2007) has concluded that the standard picture of an outer core of a neutron star consisting of coexisting superfluid neutrons and type II superconducting protons is inconsistent with the existence of long-period precession. Free precession then means that the neutron vortices at the inner crust must not be pinned, in agreement with similar conclusions reached by Shaham (1977) and Jones \& Anderson (2001).

Acknowledgements. We acknowledge the support through DFG grant St 173/31 and the corresponding RFBR grant 06-02-16025, as well as significant contributions to the data analysis by L. Rodina. We thank the anonymous referee for pointing to the question of shifts in pulse arrival time. We are thankful to $\mathrm{J}$. Trümper for suggesting that we emphasize the general importance of free precession to the physics of the neutron star interior.

\section{References}

Alpar, A., \& Ögelman, H. 1987, A\&A, 185, 196 Anderson, P. W., \& Itoh, N. 1975, Nature, 256, 25 
Brecher, K. 1972, Nature, 239, 325

Bisnovatyi-Kogan, G. S., \& Kahabka, P. 1993, A\&A, 267, L43

Bisnovatyi-Kogan, G. S., Mersov, G. A., \& Scheffer, E. K. 1989, A\&A, 221, L7

Coburn, W., Heindl, W. A., Rothschild, R. E., et al. 2002, ApJ, 580, 394

Cutler, C., Ushomirsky, G., \& Link, B. 2003, ApJ, 588, 975

Deeter, J. E., Scott, D. M., Boynton, P. E., et al. 1998, ApJ, 502, 802

Gerend, D., \& Boynton, P. E. 1976, ApJ, 209, 562

Giacconi, R., Gursky, H., Kellog, E., et al. 1973, ApJ, 184, 227

Gruber, D. E., Matteson, J. L., Nolan, P. L., et al. 1980, ApJ, 240, L127

Haberl, F., Turolla, R., de Vries, C. P., et al. 2006, A\&A, 451, L17

Jones, D. I., \& Anderson, N. 2001, MNRAS, 324, 811

Ketsaris, N. A., Kuster, M., Postnov, K. A., et al. 2000, in Proc. Hot points in Astrophysics, Joint Inst. for Nucl. Res., Dubna, 192

Klein, F., \& Sommerfeld, A. 1910, Theorie des Kreisels (Leibzig: Teubner)

Klochkov, D., Shakura, N., Postnov, K., et al. 2006, Astron. Lett., 32, 804

Klochkov, D., Staubert, R., Postnov, K., et al. 2008, A\&A, 482, 907

Lamb, D. Q., Lamb, F. K., Pines, D., \& Shaham, J. 1975, ApJ, 198, L21

Levin, Y., \& D’ Angelo 2004, ApJ, 613, 1157

Link, B. 2007, Ap\&SS, 308, 435

Link, B., \& Epstein, R. I. 2001, ApJ, 556, 392

Postnov, K. A., Prokhorov, M. E., \& Shakura, N. I. 1991, in NAS-NRC, High Energy Astrophysics, American and Soviet Perspectives, 307

Postnov, K. 2004, Topics in X-ray Astronomy (Tübingen), see

http:astro.uni-tuebingen.de/staubert65/talks/postnov.pdf

Ruderman, M. A. 1970, Nature, 225, 838
Schandl, S., \& Meyer, F. 1994, A\&A, 289, 149

Scott, D. M., Leahy, D. A., \& Wilson, R. B. 2003, ApJ, 539, 392

Sedrakian, A., Wasserman, I., \& Cordes, J. M., ApJ, 524, 341

Shaham, J. 1977, ApJ, 214, 251

Shakura, N. I. 1988, in Physics of Neutron Stars, Liningrad, 34 (in Russian), and in Physics of Neutron Stars, ed. A. M. Kaminker (Commack, N.Y.: Nova Science Publisher), 1995, 55 (English translation)

Shakura, N. I., Postnov, K., \& Prokhorov, M. 1998, A\&A, 331, L37

Shakura, N. I., Prokhorov, M. E., Postnov, K. A., \& Ketsaris, N. A. 1999, A\&A, 348,917

Soong, Y., Gruber, D. E., Peterson, L. E., \& Rothschild, R. E. 1990, ApJ, 348, 634

Staubert, R., Bezler, M., \& Kendziorra, E. 1983, A\&A, 117, 215

Staubert, R., Schandl, S., \& Wilms, J. 2000, in Proc. 4th COMPTON Symp., AIP Conf. Proc., 510, 153

Staubert, R., Schandl, S., Klochkov, D., et al. 2006, in Proc. The transparent Milky Way: a perspective for MIRAX, AIP Conf. Proc., 840, 65

Still, M., \& Boyd, P. 2004, ApJ, 606, L135

Still, M., \& Jurua, E. 2006, BAAS, 38, 339

Trümper, J., Kahabka, P., Ögelmann, H., Pietsch, W., \& Voges, W. 1986, ApJ, 300, L63

Tananbaum, H., Gursky, H., Kellog, E. M., et al. 1972, ApJ, 174, L143

Wasserman, I. 2003, MNRAS, 341, 1020

Wilms, J., Ketsaris, N. A., Postnov, K. A., et al. 2003, Isvestiya Akademii Nauk, Ser. Fizicheskaya, 67, 310 (in Russian) 